\title{
DELTAS OF HOCHSCHILD DIMENSION ONE
}

\author{
CHARLES CHING-AN CHENG
}

\begin{abstract}
A theorem of Mitchell says that free categories have Hochschild dimension $<1$. The converse is shown to be true for all deltas (i.e. small skeletal categories whose only endomorphisms are the identities).
\end{abstract}

Throughout $K$ will denote a nonzero commutative ring with identity, and $\mathfrak{N}$ will be the category of $K$-modules. Let $\mathbf{C}$ be a small category, and let $K \mathbf{C}(p, q)$ be the free $K$-module on the set $\mathbf{C}(p, q)$ of morphisms from $p$ to $q$. Then $K \mathbf{C}$ can be regarded as an object of the functor category $\Re^{\mathbf{C}^{\circ} \times \mathbf{C}}$, and its projective dimension is called the $K$-Hochschild dimension of $\mathbf{C}\left(\operatorname{dim}_{K} \mathbf{C}\right)$. Clearly $\operatorname{dim}_{K} \mathbf{C}=\operatorname{dim}_{K} \mathbf{C}^{\text {op }}$. In [2, p. 62] it is shown that if $D \in \mathfrak{T}^{\mathbf{C}}$, then

$$
\text { pd } D \leqslant \operatorname{dim}_{K} C+\sup _{p \in C} \operatorname{pd} D(p) \text {, }
$$

where pd denotes projective dimension. In particular, if $D$ has free $K$-modules as values, then $\operatorname{pd} D \leqslant \operatorname{dim}_{K} C$. When $C$ is a group, $\operatorname{dim}_{K} C$ is the same as the $K$-cohomological dimension of the group [1, p. 195]. Therefore by Stallings [3] and Swan [4] we know that $\operatorname{dim}_{z} C \leqslant 1$ if and only if $C$ is free as a group. In this paper we show that if $\mathbf{C}$ is a delta (i.e. a small skeletal category whose only endomorphisms are the identities) then $\operatorname{dim}_{K} C \leqslant 1$ if and only if $\mathbf{C}$ is the free category generated by a directed graph. This completes a theorem of Mitchell [2, p. 151], who established it in case $\mathbf{C}$ is either a weak delta or a partially ordered set.

Henceforth $\mathbf{C}$ will denote a delta. If $\alpha$ is a morphism in $\mathbf{C}$ then we denote its domain and codomain by $\operatorname{dom} \alpha$ and $\operatorname{cod} \alpha$, respectively. The length of $\alpha$ is defined to be $\sup \left\{k \mid \alpha=\alpha_{1} \alpha_{2} \cdots \alpha_{k}, \alpha_{i} \neq 1\right\}$. If $D \in \mathfrak{N}^{\mathrm{C}}$ then we denote the image of $D(\alpha)$ by $\alpha D(p)$. If $p$ and $q$ are objects of $\mathbf{C}$, define $p \leqslant q$ if $\mathbf{C}(p, q) \neq \varnothing$. Because $\mathbf{C}$ is skeletal and all endomorphisms are identities, it follows that this is a partial order. For each $p \in \mathbf{C}$ let $S_{p}: \mathfrak{T} \rightarrow \mathfrak{N}^{\mathrm{C}}$ denote the left adjoint of the $p$ th evaluation functor $D \mapsto D(p)$. Then $S_{p}$ is given explicitly by

$$
S_{p}(A)(q)=\underset{\mathbf{C}(p, q)}{\bigoplus} A .
$$

Note that $S_{p}$ preserves projectives since it has an exact right adjoint.

LEMMA 1. Let $D \in \mathfrak{T C}^{\mathrm{C}}$ be projective. If $\alpha_{i}: p_{i} \rightarrow q, i=1,2$, are morphisms

Received by the editors November 19, 1976.

AMS (MOS) subject classifications (1970). Primary 18G20; Secondary 18B99.

Key words and phrases. Delta, Hochschild dimension.

(c) American Mathematical Society 1978 
in $\mathbf{C}$, then

$$
\alpha_{1} D\left(p_{1}\right) \cap \alpha_{2} D\left(p_{2}\right)=\sum \alpha_{1} \beta_{1} D\left(\operatorname{dom} \beta_{1}\right),
$$

where the sum is indexed by all $\beta_{1}$ with codomain $p_{1}$ such that there exists $\beta_{2}$ making the following diagram commutative.

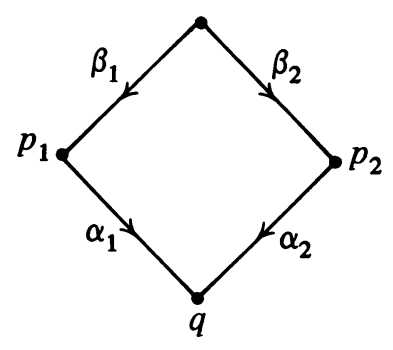

Proof. The right side of (2) is always contained in the left side, and the inclusion is a natural transformation of functors of the variable $D$. It follows that coproducts and retracts of objects satisfying (2) also satisfy (2). Clearly $S_{p}(A)$ satisfies (2) for all $A \in \mathfrak{K}$ and $p \in \mathbf{C}$. Consider the epimorphism

$$
\pi: \bigoplus_{p \in \mathrm{C}} S_{p}(D(p)) \rightarrow D
$$

where the $p$ th coordinate of $\pi$ is induced by the identity map. Since $D$ is projective, $\pi$ splits. Hence $D$, as a retract of a coproduct whose factors satisfy (2), also satisfy (2).

LemMa 2. Suppose $\operatorname{dim}_{K} \mathbf{C} \leqslant 1$ and the diagram below is commutative. If either $\alpha_{1}$ and $\alpha_{2}$ have length one or $\operatorname{dom} \alpha_{1}=\operatorname{dom} \alpha_{2}$, then $\alpha_{1}=\alpha_{2}$.

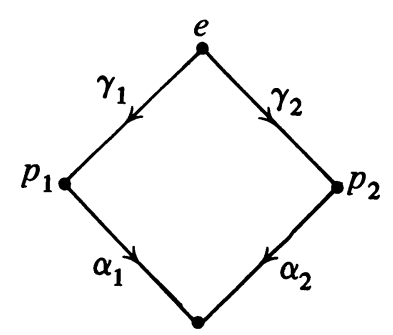

Proof. Let $E=S_{e}(K)$. Define $D \in \mathfrak{R}^{\mathrm{C}}$ as follows:

$$
\begin{array}{rlrl}
D(p) & =E(p) & & \text { if there exists } \beta_{i}: p \rightarrow p_{i}, i=1,2, \\
& & \text { with } \alpha_{1} \beta_{1}=\alpha_{2} \beta_{2}, \\
& =0 & & \text { otherwise. }
\end{array}
$$

Consider the short exact sequence $0 \rightarrow N \rightarrow E \rightarrow^{\pi} D \rightarrow 0$ where $\pi_{p}$ is the identity map whenever possible. Since $\operatorname{dim}_{K} C \leqslant 1$, we have pd $D \leqslant 1$. Therefore since $E$ is projective, $N$ is projective. By Lemma 1, we have

$$
\alpha_{1} N\left(p_{1}\right) \cap \alpha_{2} N\left(p_{2}\right)=\sum_{\beta_{1}} \alpha_{1} \beta_{1} N\left(\operatorname{dom} \beta_{1}\right)=0 .
$$


If $\alpha_{1} \neq \alpha_{2}$ and if either $\alpha_{1}$ and $\alpha_{2}$ have length one or $\operatorname{dom} \alpha_{1}=\operatorname{dom} \alpha_{2}$, then there exists no commutative square of the form

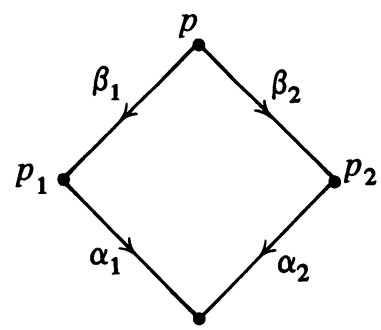

where $p$ is either $p_{1}$ or $p_{2}$. Here we used the fact that all endomorphisms of $\mathbf{C}$ are identities. Hence $N\left(p_{i}\right)=E\left(p_{i}\right), i=1,2$. Since $E$ is projective,

$$
\begin{aligned}
\alpha_{1} N\left(p_{1}\right) \cap \alpha_{2} N\left(p_{2}\right) & =\alpha_{1} E\left(p_{1}\right) \cap \alpha_{2} E\left(P_{2}\right) \\
& =\sum_{\beta_{1}} \alpha_{1} \beta_{1} E\left(\operatorname{dom} \beta_{1}\right) \supseteq \alpha_{1} \gamma_{1} E(e) \neq 0 .
\end{aligned}
$$

This contradicts (3).

We need the following result [2, Corollary 36.11].

LemMa 3. If $\operatorname{dim}_{K} \mathbf{C} \leqslant 1$, then every morphism of $\mathbf{C}$ is a composite of morphisms of length one.

Theorem 4. $\operatorname{dim}_{K} \mathbf{C} \leqslant 1$ if and only if $\mathbf{C}$ is free.

Proof. The "if" part is known for any small category $\mathbf{C}$ [2, Corollary 28.3].

Suppose $\operatorname{dim}_{K} \mathbf{C} \leqslant 1$ and suppose there is a morphism that has two representations as composites of morphisms of length one, say, $x_{1} x_{2} \cdots x_{m}$ $=y_{1} y_{2} \cdots y_{n}, m<n$. We will show that $m=n$ and $x_{i}=y_{i}, i=1$, $2, \ldots, m$. This is clearly true if $m=1$. Using the first part of Lemma 2 we see that $x_{1}=y_{1}$. Then, by the dual of the second part of Lemma 2, $x_{2} \cdots x_{m}=y_{2} \cdots y_{n}$. Hence, by induction, $m=n$ and $x_{i}=y_{i}, 2 \leqslant i \leqslant m$.

ACKNowledGement. I would like to thank the referee for helping to simplify the exposition of the paper.

\section{REFERENCES}

1. H. Cartan and S. Eilenberg, Homological algebra, Princeton Univ. Press, Princeton, N. J., 1956.

2. B. Mitchell, Rings with several objects, Advances in Math. 8 (1972), 1-161.

3. J. R. Stallings, On torsion free groups with infinitely many ends, Ann. of Math. (2) 88 (1968), 312-334.

4. R. G. Swan, Groups of cohomological dimension one, J. Algebra 12 (1969), 585-610.

Department of Mathematics, OAKLAND University, Rochester, Michigan 48063 